\title{
Creating an Intensive Care Unit From a Postanesthesia Care Unit for the COVID-19 Surge at the Veterans Affairs Ann Arbor Healthcare System
}

\author{
Mary Jarzebowski, MD; Tom Curran, MD, MBA; Megan Dorsey, MD; William Cederquist, MD; Dru Claar, MD; \\ Elisa Derrig, CRNA; Weston Dick, BSME; Katrina Push, RN, BSN, CPAN; Mark Hausman, MD; and Tamar Lake, MD
}

Objectives: To prepare for the predicted surge of patients with COVID-19 in Southeast Michigan, the US Department of Veterans Affairs Ann Arbor Healthcare System engineered, built, and staffed a 12-bed intensive care unit (ICU) from the existing postanesthesia care unit (PACU).

Observations: Considerations including floor planning, reversal of airflow, strict airborne precautions, sealing off a dedicated nursing station, and developing an infection control plan in an open care unit. A staffing model was created that included anesthesiologist intensivists, advanced practice providers, residents, certified registered nurse anesthetists, and perioperative nurses working alongside ICU trained nurses. Challenges arose in infection control, communication, mechanical ventilation using anesthesia machines, providing renal replacement therapy, and maintaining patient privacy in an open unit.

Conclusions: This article describes the setup, challenges, and solutions that allowed the creation of the PACU-ICU to help serve veterans and civilians during a time of unprecedented strain on the health care system due to COVID-19.
Author information is available at the end of the article.

Correspondence: Mary Jarzebowski (mjarzebo@med.umich.edu)

Fed Pract. 2020;37(8):348-353 doi:10.12788/fp.0020
$\mathrm{T}$ he rise in prevalence of the community spread of coronavirus disease 2019 (COVID-19) in the US in early March 2020 led to hospital systems across the country preparing for an increase in critically ill patients. ${ }^{1}$ The US Department of Veterans Affairs (VA) Ann Arbor Healthcare System (VAAAHS) anticipated an increased census of veterans who would need hospital admission for severe COVID-19 as well as the potential need to receive patients from community hospitals in Southeast Michigan, the location of one of the worst outbreaks in the US at that time. ${ }^{2}$

Through the facility's incident command center, a hospital operations group identified the postanesthesia care unit (PACU) as a space to convert to an intensive care unit (ICU) for patients with COVID-19 needing mechanical ventilation. Other hospitals throughout the world have created similar makeshift ICUs to help care for the surge of patients with COVID-19, recognizing the high level of monitoring and resources available in the perioperative setting. ${ }^{3-5}$ These ICUs have been successfully created in operating rooms, ${ }^{3}$ recovery rooms, ${ }^{5}$ and procedural settings. ${ }^{4}$

Between March 27, 2020 and April 25, 2020 , a great multidisciplinary effort enabled the VAAAHS PACU-ICU to care for critically ill veterans with COVID-19 from Southeast Michigan as well as civilian transfers from overwhelmed neighboring community hospitals. This article will discuss planning considerations, including facility preparation, equipment, and staffing models. The unique challenges faced in managing an open-plan surge-capacity ICU also will be discussed as well as the solutions that were enacted.

\section{METHODS}

\section{Hospital Preparation}

Maintaining a 2-zone model in which patients with COVID-19 and without COVID19 could be cared for separately was of major importance. The VAAAHS traditional ICU was converted into a 16-bed COVID-19 ICU and staffed by the Pulmonary Critical Care Service. A separate wing of the hospital was converted into a 19-bed non-COVID-19 ICU, which also was staffed by the Pulmonary Critical Care Service that increased its staffing of residents, fellows, and attending physicians to meet the increasing clinical demands. Elective major surgery cases were postponed, and surgeons managed the care of postoperative surgical ICU patients. This arrangement allowed the existing 4 anesthesiologist intensivists to staff the PACU COVID-19 ICU. 
Considerations, including space requirements, staffing, equipment, infection control requirements, and ability for facilities to engineer a negative pressure space were factored into the decision to convert the PACU to an additional 12-bed ICU. This effectively tripled the VAAAHS ICU capacity, enabling patient transfers from the John D. Dingell VA Medical Center in Detroit, Michigan, which was being impacted by a surge of cases in Detroit. In addition, this allowed for the opening of the hospital for both COVID-19 and nonCOVID-19 ICU transfers from hospitals in Southeast Michigan in order to fulfill the fourth VA mission to provide care and support to state and local communities for emergency management, public health, and safety.

\section{PACU Preparation}

PACU was selected as an overflow ICU due to its open floor plan, allowing patients on ventilators to be seen from a central nursing station. This would allow for the safe use of ventilators without central alarm capabilities (especially anesthesia machines). Given the risk of a circuit disconnect, all ventilators without central alarm capabilities needed to be seen and heard within the space to ensure patient safety.

Facilities Management was able to construct temporary barriers with vinyl covered sheetrock and plexiglass to partition the central nursing workstation from the patient area in a U-shape (Figure 1). The patient area was turned into a negative pressure space where strict airborne precautions could be observed. Although the air handling unit serving this space is equipped with high efficiency particulate air (HEPA) filters, it was mechanically manipulated to ensure that all air coming from the space was discharged through exhaust and not recirculated into another occupied space within the hospital. Total air exchange rates were measured and calculated for both the positive and negative spaces to ensure they met or exceeded at least 6 air changes per hour, as recommended by Occupational Safety and Health Administration guidance. ${ }^{6,7} \mathrm{~A}$ differential pressure indicator was installed to provide staff with the ability to monitor the pressure relationship between the 2 spaces in real time.

Twelve patient care beds were created. A traditionally engineered airborne infection
FIGURE 1 PACU-ICU Floor Plan

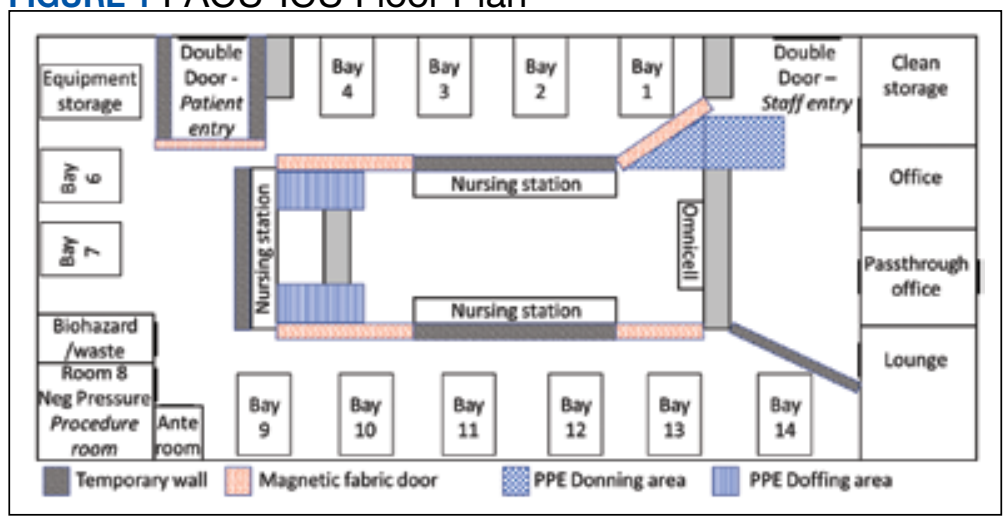

Abbreviations: ICU, intensive care unit; PACU, postanesthesia care unit, PPE, personal protective equipment.

isolation room in PACU served as a procedure room for aerosol-generating procedures, especially intubation, extubation, use of high-flow nasal cannula, and tracheostomy placement. Strict airborne precautions were taken within the patient area. The area inside the nursing station was positively pressurized to allow for surgical masks only to be required for the comfort of health care workers (Figure 2). A clear donning and doffing workflow was created for movement between the nursing area and the patient care area.

\section{Personal Protective Equipment}

Personal protective equipment (PPE) was of paramount importance in this open care unit. Airborne precautions were used in the entire patient care area. Powered airpurifying respirators (PAPRs) were used when possible to conserve the supply of N95 masks. Each health care worker was issued a reusable PAPR hood, which was cleaned by the user after each use by wiping the exterior of the entire hood with virucidal wipes. The brand and active ingredient of the virucidal wipes varied by availability of supplies, but the "virus kill time" was clearly labeled on each container. Each health care worker had a paper bag for storing his or her PAPR hood between usage to allow drying and ventilation. PAPR units were charged in between uses and shared by all clinical staff. Two layers of nonsterile gloves were worn.

Because of the open care area, attention had to be given to adhere to infection control policies if health care workers wanted to care for multiple patients while in the area. A new gown was placed over the existing gown, and 
FIGURE 2 Central Nursing Station Before (left) and After (right) Construction of Temporary Walls

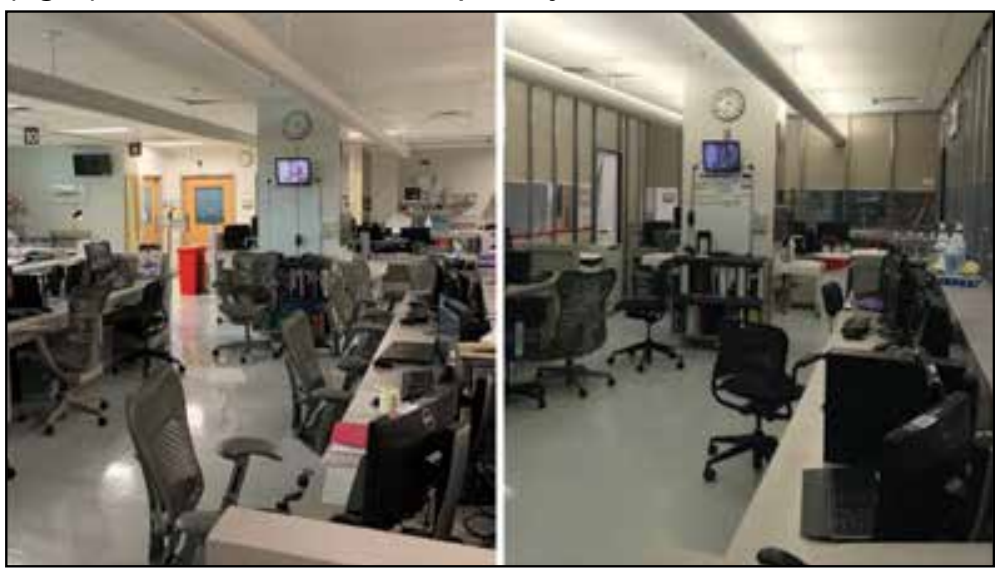

the outer layer of gloves was removed. The under layer of gloves was then sanitized with hand sanitizer, and a new pair of outer gloves was then worn.

\section{Equipment}

Much of the ICU-level equipment needed was already present within the operating room (OR) area. Existing patient monitors were used and connected to a central monitoring station present in the nurses station. Relevant contents of the ICU storage room were duplicated and placed on shelves in the patient care area. Out-of-use anesthesia carts were used for a dedicated COVID-19 invasive line cart. A designated ultrasound with cardiac and vascular access probes was assigned to the PACU-ICU. Anesthesia machines were brought into the PACU-ICU and prepared with viral filters in line to prevent contamination of the machines, in keeping with national guidance from the American Society of Anesthesiologists and Anesthesia Patient Safety Foundation. ${ }^{8}$

MULTIDISCIPLINARY STAFFING MODEL With the reduced surgical and procedural case load due to halting nonemergent operations, the Anesthesiology and Perioperative Care Service was able to staff the PACU-ICU with critical care anesthesiologists, nurse anesthetists, residents, and PACU and procedural nurses without hindering access to emergent surgeries. A separate preoperative area was maintained with an 8-bed capacity for both preoperative and postoperative management of non-COVID-19 surgical patients.
The staffing model was designed using guidance on the expansion of ICU staffing with non-ICU resources from the Society of Critical Care Medicine as well as local guidance on appropriate nursing ratios (Figure 3). ${ }^{9}$ Given the high acuity and dynamic nature of COVID-19 coupled with the unique considerations that exist using anesthesia machines as long-term ICU ventilators, 24hour inhospital attending intensivist coverage was provided in the ICU by 4 critical care anesthesiologists who rotated between 12-hour day and night shifts. The critical care anesthesiologists led a team of anesthesiology and surgery residents and ICU advanced practice providers dedicated solely to the PACU-ICU. Non-ICU anesthesiologists helped with procedures such as intubation and invasive line placement and provided coverage of the ICU patients during signout and rounding. Certified registered nurse anesthetists (CRNAs) performed intubations and helped offload respiratory therapists (one of the resources most in shortage) by managing and weaning ventilators and were instrumental in prone positioning of patients. Dedicated ICU nurses were deployed every shift to oversee the unit and act as a resource to the PACU nurses. Fortunately, many PACU nurses had prior ICU training and experience, and nurses from outpatient areas also were recruited to help with patient care. Together, they provided direct patient care. OR nurses assisted with delivering supplies, medications and transporting specimens to the laboratory, as no formal hospital tube station was present in the PACU.

Because of the open-unit setting, nurses practiced bundled care and staggered their turns in the patient care area. For example, a nurse who entered to administer medication to patient $\mathrm{A}$, could then receive communication to check the urine output for patient $\mathrm{B}$ and do so without completely doffing and redonning. This allowed preservation of PPE and reduced time in PPE for the health care providers (HCPs).

A scheduled daily meeting included staff from PACU-ICU; Medical ICU (MICU), which also treated patients with COVID-19; and the Palliative Care Service (Figure 4). Patients with single-organ failure were preferentially sent to PACU-ICU, as the ability to do renal replacement therapy (RRT) in an 
open unit proved difficult. The palliative care team and VAAAHS social workers assisted both MICU and PACU-ICU with communicating with patients' families, which provided a great help during a clinically demanding time. Physical therapists increased their staffing of the ICU to specifically help with mobilization of patients with COVID-19 and acute respiratory distress syndrome, given the prolonged mechanical ventilation courses that were seen. Other consulting services frequently involved included infectious disease and nephrology.

\section{CHALLENGES AND SOLUTIONS}

Communication between staff located within the patient area and staff located in the nursing station was difficult given the loud noise generated by a PAPR and the plexiglass walls that separated the areas. Multiple techniques were attempted to overcome this. Dry erase boards were placed within the space to facilitate requests, but these were found to be time consuming. Two-way radios worked well if the users were wearing N95s but were harder to communicate when users were wearing PAPRs. Baby monitors were purchased to facilitate 2-way communication and were useful at times although quieter than desired. Vocera B3000N Communication Badges, which were already utilized in the perioperative period at the facility, could be utilized underneath PPE and were ultimately the best form of clear communication between staff within the patient care area and outside the negative pressure zone. In accordance with company guidance, these mobile devices were cleaned with virucidal wipes after use. ${ }^{10}$

Communication with patients' families was critically important. The ICU team, palliative care team, or social workers made daily telephone calls to family members. The facility telehealth coordinator provided a designated tablet device to enable the intensivists to video conference with the patients' families at bedside, utilizing virtual care manager appointments. This allowed families to see and interact with their loved ones despite the prohibition of family visitors. Every effort was made to utilize video calling daily; however, clinical demands as well as Internet and technological constraints from individual family members intermittently precluded video calls.
FIGURE 3 PACU-ICU Staffing Model, Utilizing Perioperative Staff to Extend ICU Resources

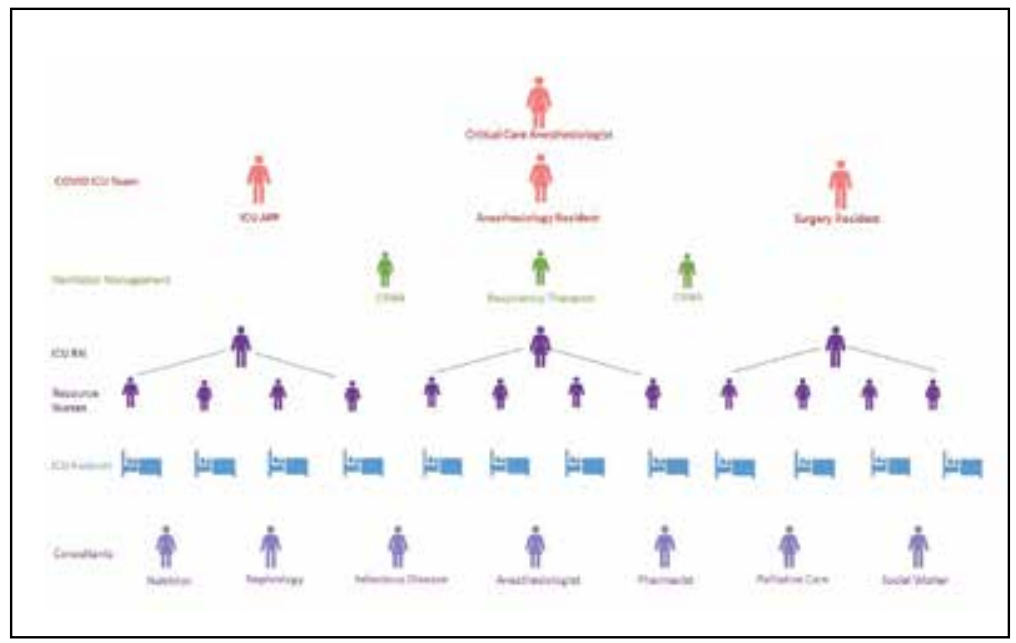

Abbreviations: APP, advanced practice provider; CRNA, certified RN anesthetist; ICU, intensive care unit; PACU, postanesthesia care unit, RN, registered nurse.

\section{Clinical Challenges}

Patients with severe COVID-19 infections requiring mechanical ventilation have proven to be exceptionally high-acuity patients with myriad organ-based complications reported. ${ }^{11}$ Specific to our PACU-ICU, we determined that it was impractical to arrange for continuous RRT given the amount of training PACU nursing staff would have required and the limited ICU nursing staff in the PACUICU. Intermittent hemodialysis required replumbing for water supply and drainage but was ultimately not required as our facility expanded the number of continuous RRT machines available, allowing all patients in the COVID-19 ICU who required RRT to stay in the 16-bed ICU. Daily communication with the MICU allowed for safe transfer of patients with imminent needs for RRT to the MICU, providing a coordinated strategy for the deployment of scarce resources across our expanded ICU footprint.

Using anesthesia machines as ICU ventilators proved challenging, despite following best practice guidance. ${ }^{8}$ Notably, anesthesia machines are not actively humidified and require very high fresh gas flows, necessitating the addition of heat moisture exchangers (HME) to the circuit. Also, viral filters were placed in the circuit to prevent machine contamination. The addition of the HME and viral filters to each circuit increased the present dead space and led to 


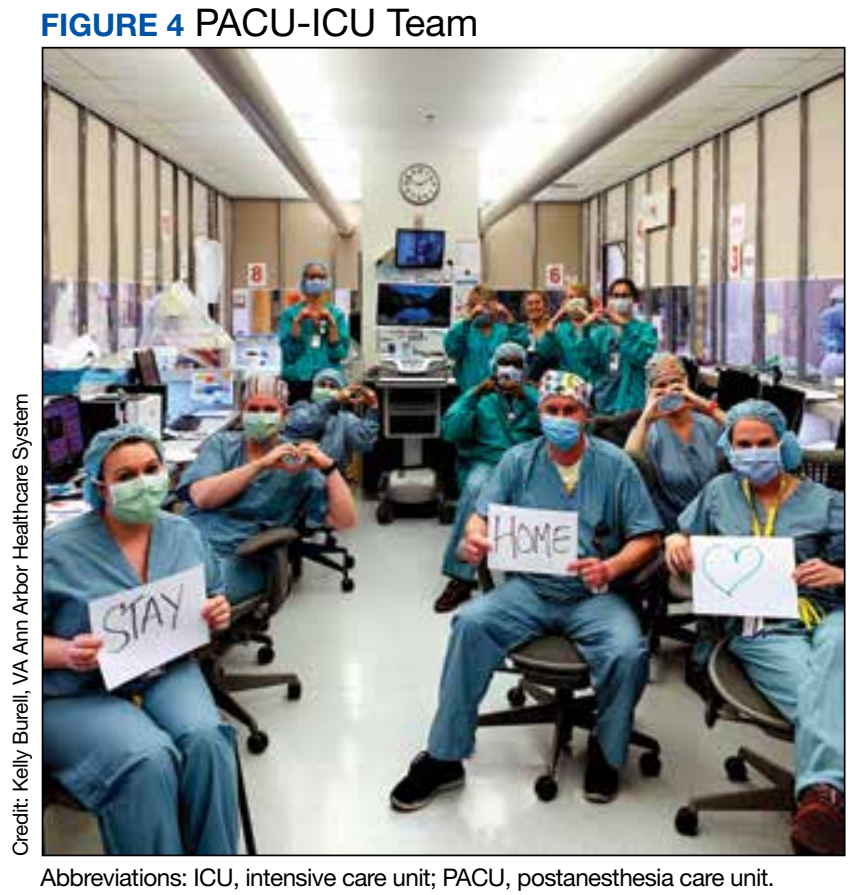

difficulty in providing adequate ventilation to patients who already may have had a high proportion of physiologic dead space. The high fresh gas flows used still seemed inadequate in preventing moisture buildup in the machine parts, necessitating frequent exchanges of viral filters, HMEs, and circuits to prevent high peak airway pressures. In addition, anesthesia machines directly sample gas from the patient's breathing circuit, creating the risk for contamination of the space. This required a reconfiguration to allow for a suction scavenging system by VAAAHS biomedical engineers. Also, anesthesia machines are not designed for long-term ventilation and have different ventilation modes compared with modern ICU ventilators. Although they were used for several patients when the PACU-ICU opened, the hospital was able to acquire additional ICU ventilators, and extensive or prolonged use of anesthesia machine ventilators was avoided.

\section{Infection Control}

The open care setting provided unique infection control issues that had to be addressed. ${ }^{12}$ The open setting allowed preservation of PPE and the ability for bundled care to be delivered easily. The VAAAHS infection control team worked closely with the ICU team to develop practices to ensure both patient and health care worker protection. Notable challenges included donning new gowns between patients when a PAPR was already being worn, leading to draping of new gowns over existing gowns when going between patients. True hand hygiene was also difficult, as health care workers did not want to completely remove gloves while in the patient care area. Layering of 2 pairs of gloves allowed the outer gloves to be removed after care of each patient, at which time alcohol gel was applied to the inner gloves, a new gown was placed over the existing gown, and a new pair of gloves was layered on top.

Although patients were intubated for long periods in the PACU-ICU, there was concern for increased risk of exposure of health care workers after extubation given the inability to contain the coughing patients within a private room. If a patient did well, they were transferred to a private room on the general medical floors within 24 hours of extubation to minimize this risk.

\section{Privacy}

The open care design meant less privacy for patients than would be provided in a private room. Curtains were drawn around patient beds as much as possible, especially for nursing care, but priority was given to visualization of the ventilator when a HCP was not present to ensure safety at all times. The majority of patients cared for in the PACU-ICU were intubated and sedated on arrival, but thankfully many were extubated. After extubation privacy in the open care area became more of an issue and may have led to more nighttime disturbances and substandard delirium prevention measures. Priority was given to expediting the transfer of these patients to private rooms on the general medical floor once their respiratory status was deemed stable.

\section{CONCLUSIONS}

The COVID-19 pandemic is truly an unprecedented event in our nation's history, which has led to the first nationwide authorization of the fourth mission of VA to provide support for national, state, and local public health. The PACU-ICU was designed, engineered, built, and staffed by perioperative HCPs through an exceptional multidisciplinary effort in a matter of days. Through 
this dedication of health care workers and staff, the VAAAHS was able to care for critically ill veterans from Southeast Michigan and serve the community during a time of overwhelming demand on the national health care system.

\section{Author affiliations}

Mary Jarzebowski, Megan Dorsey, and William Cederquist are Staff Physicians; Tom Curran is Director, Surgical Intensive Care Unit; Dru Claar is Director, Medical Intensive Care Unit; Elisa Derrig is Chief CRNA, Section of Anesthesiology; Weston Dick is Chief, Facilities Management Service; Katrina Push is a Nurse Manager, Post-Anesthesia Care Unit; Mark Hausman is Chief of Staff; and Tamar Lake is Chief, Section of Anesthesiology and Perioperative Care; all at the Veterans Affairs Ann Arbor Healthcare System in Michigan. Mary Jarzebowski is a Clinical Assistant Professor; Tom Curran, Megan Dorsey, and William Cederquist are Clinical Instructors; Mark Hausman is an Assistant Professor; all in the Department of Anesthesiology, University of Michigan. Dru Claar is a Clinical Assistant Professor, Department of Pulmonary and Critical Care Medicine at the University of Michigan. Mark Hausman is Assistant Dean for Veterans Affairs, University of Michigan Medical School.

\section{Acknowledgments}

The authors thank the outstanding team of administrators, engineers, physical therapists, pharmacists, nurses, advanced practice providers, CRNAs, respiratory therapists, and physicians who made it possible to respond to our veterans' and our community's needs in a time of unprecedented demand on our health care system. A special thank you to Eric Deters, Chief Strategy Officer; Brittany McClure, ICU Nurse Manager; and Mark Dotson, Chief Supply Chain Officer. It was a privilege to serve on this mission together.

\section{Author disclosures}

The authors report no actual or potential conflicts of interest with regard to this article.

\section{Disclaimer}

The opinions expressed herein are those of the authors and do not necessarily reflect those of Federal Practitioner, Frontline Medical Communications Inc., the US Government, or any of its agencies. This article may discuss unlabeled or investigational use of certain drugs. Please review the complete prescribing information for specific drugs or drug combinations - including indications, contraindications, warnings, and adverse effects-before administering pharmacologic therapy to patients.

\section{References}

1. Murray CJL; IHME COVID-19 Health Service Utilization Forecasting Team. Forecasting COVID-19 impact on hospital bed-days, ICU-days, ventilator days and deaths by US state in the next 4 months. https://www.medrxiv.org /content/10.1101/2020.03.27.20043752v1.full.pdf. Accessed July 17, 2020.

2. Johns Hopkins University and Medicine. Coronavirus resource center. https://coronavirus.jhu.edu/data/state -timeline/new-confirmed-cases/michigan. Updated July 17, 2020. Accessed July 17, 2020.

3. Mojoli F, Mongodi S, Grugnetti G, et al. Setup of a dedicated coronavirus intensive care unit: logistical aspects. Anesthesiology. 2020;133(1):244-246. doi:10.1097 /ALN.0000000000003325

4. Peters AW, Chawla KS, Turnbull ZA. Transforming ORs into ICUs. N Engl J Med. 2020;382(19):e52. doi:10.1056/NEJMc2010853

5. Lund E, Whitten A, Middleton R, Phlippeau N, Flynn DN. Converting peri-anesthesia care units into COVID-19 critical care units: one community hospital's response. Anesthesiology News. April 30, 2020. https://www .anesthesiologynews.com/Online-First/Article/04-20 /Converting-Peri-Anesthesia-Care-Units-Into-COVID -19-Critical-Care-Units/58167. Accessed July 14, 2020.

6. American Institute of Architects. Guidelines for Design and Construction of Hospitals and Healthcare Facilities. Washington, DC: American Institute of Architects Press; 2001.

7. Garner JS. The CDC Hospital Infection Control Practices Advisory Committee. Am J Infect Control. 1993;21(3):160162. doi:10.1016/0196-6553(93)90009-s

8. American Society of Anesthesiologists. APSF/ASA Guidance on Purposing Anesthesia Machines as ICU Ventilators. https://www.asahq.org/in-the-spotlight /coronavirus-covid-19-information/purposing-anesthesia -machines-for-ventilators. Updated May 7, 2020. Accessed July 14, 2020.

9. Halpern NA, Tan KS. United States Resource Availability for COVID-19. https://sccm.org/getattachment/Blog /March-2020/United-States-Resource-Availability-for -COVID-19/United-States-Resource-Availability-for -COVID-19.pdf. Updated May 12, 2020. Accessed July $14,2020$.

10. Vocera. Vocera devices and accessories cleaning guide. http://pubs.vocera.com/device/vseries/production/docs /vseries_device_cleaning_guide.pdf. Updated June 24, 2020. Accessed July 14, 2020.

11. Poston JT, Patel BK, Davis AM. Management of Critically III Adults With COVID-19 [published online ahead of print, 2020 Mar 26]. JAMA. 2020;10.1001/jama.2020.4914. doi:10.1001/jama.2020.4914

12. O'Connell $\mathrm{NH}$, Humphreys $\mathrm{H}$. Intensive care unit design and environmental factors in the acquisition of infection. J Hosp Infect. 2000;45(4):255-262. doi:10.1053/jhin.2000.0768 